\title{
Early and interdisciplinary physiotherapy in a patient in the intensive care unit with a bronchopulmonary fistula after thoracic fenestration: a case report
}

\begin{abstract}
The aim of this study is to present the effects of the medical team's therapy with particular focus on early physiotherapy in a patient subjected to elective pneumonectomy due to pleural empyema and chest fenestration. Our patient was 48 years old and underwent an elective pneumonectomy due to pleural empyema and cicatrization atelectasis as a result of complications. A lack of lung recoil after chest fenestration was the indication to transfer the patient to the intensive care unit (ICU) from the operating theater. Respective rehabilitation is necessary to restore the function of the respiratory system. It should be an integral part of the treatment plan, must be implemented in a timely manner, and should continue until the patient's full recovery. This paper presents the effects of the medical team's work with the emphasis on early physiotherapy in a patient in the ICU that resulted in a shorter hospitalization and a full return to functional capacity.
\end{abstract}

Key words: bronchopulmonary fistula, ICU, physiotherapy, stimulation of breathing, thoracic fenestration

Adv Respir Med. 2020; 88: 450-453

\section{Introduction}

The intensive care unit (ICU) is a common place for patients to stay after thoracic surgery when they are in a life-threatening condition. The process of treating patients in the ICU requires holistic management from the entire medical team. Operated patients are often exposed to prolonged immobilization. Early rehabilitation is safe and helps to reduce physical and psychological complications in patients. The priority is to improve the patient's critical status caused by potentially reversible failure of bodily systems. Holistic therapy is an interdisciplinary specialty in clinical medicine which also includes physiotherapy. The presented case confirms that personalized therapy has a significant impact on the improvement of treatment results while patients return to functional capacity.

\section{Case report}

A male patient aged 48 had elective surgery performed due to right pleural empyema. The patient demonstrated protein-caloric wasting (body mass index: 17.36). He underwent pleural decortication, right-sided pneumonectomy due to cicatrization atelectasis with pulmonary gangrene (TBC) with lack of recoil, and fenestration of the chest on the right side. The anterolateral sections of ribs III-IX were removed. The procedure was complicated by large blood loss $(2500 \mathrm{~mL})$ and hemorrhagic shock. The patient under, the influence of general anesthetics, was transferred from the Thoracic Surgery Operating Theater to the ICU.

Physiotherapy in the patient with a bronchopleural fistula after thoracic fenestration was introduced on the $5^{\text {th }}$ day. The early implementation of the process allowed to limit the iatrogenic effects of immobilization. The process of physiotherapy

Address for correspondence: Justyna Wyszyńska, Institute of Health Sciences, Medical College of Rzeszów University, Rzeszów, Poland; e-mail: justyna.wyszynska@onet.pl DOI: 10.5603/ARM.a2020.0128

Received: 22.04.2020

Copyright (C) 2020 PTChP

ISSN 2451-4934 
in the critically ill patient was personalized and adapted to his health condition. The management covered three comprehensive domains:

- preservation of the full range of motion in the joints and prevention of contractures;

- counteracting muscle weakening and muscle wasting;

— improvement of circulatory and respiratory function.

Resection of the lung parenchyma was, in this case, an elective treatment. A proper rehabilitation was necessary to restore efficient functioning of the respiratory system. The patient, who was in a medium-severe condition, conscious, and alert and oriented, was ventilated and infused with Levonor solution to assist circulation. The prolonged state of hypokinesia, which negatively affected the anatomy of the human body, was countered with personalized kinesiotherapy. Passive exercise and functional positioning counteracted the decrease of muscle strength while, at the same time, proprioceptive stimulation influenced the central nervous system. Buerger's exercises were also introduced covering a three-minute rotation of lower limb positioning in the following order: passive ischaemia, hyperemia, and rest.

On the $10^{\text {th }}$ day, bronchoaspiration was performed and small amounts of mucopurulent secretion were sucked out until the stump of the bronchi was sealed. Fully assisted partial verticalization was performed and a normal orthostatic change in vital parameters was noted. The effects came unexpectedly fast. From the $11^{\text {th }}$ day on, rehabilitation could be performed in the sitting position. On the $12^{\text {th }}$ day of the ICU stay, morphine was discontinued and assisted ventilation was withdrawn leaving the patient on passive therapy with nasal cannula $6 \mathrm{~L} / \mathrm{min}$. Exercises based on symmetrical pelvic movements in closed chains for the limbs were introduced in which the head and trunk are extended to enhance the sitting position during exercises of the shoulder girdle on the right side. In order to maintain proper ventilation, stimulation of respiration using different pathways was used. On the $14^{\text {th }}$ day, the patient was able to maintain the stabilization of the lower trunk independently in a position with feet resting and stabilized on the ground. Achieving a higher position allowed for the inclusion of exercises enhancing the muscular strength of the lower limbs using a bedside cycle ergometer (Figure 1). The patient was verticalized every day. On the $15^{\text {th }}$ day, tracheostomy was performed due to a need for prolonged ventilation. The patient was ventilated again. In addition, the therapist introduced man-

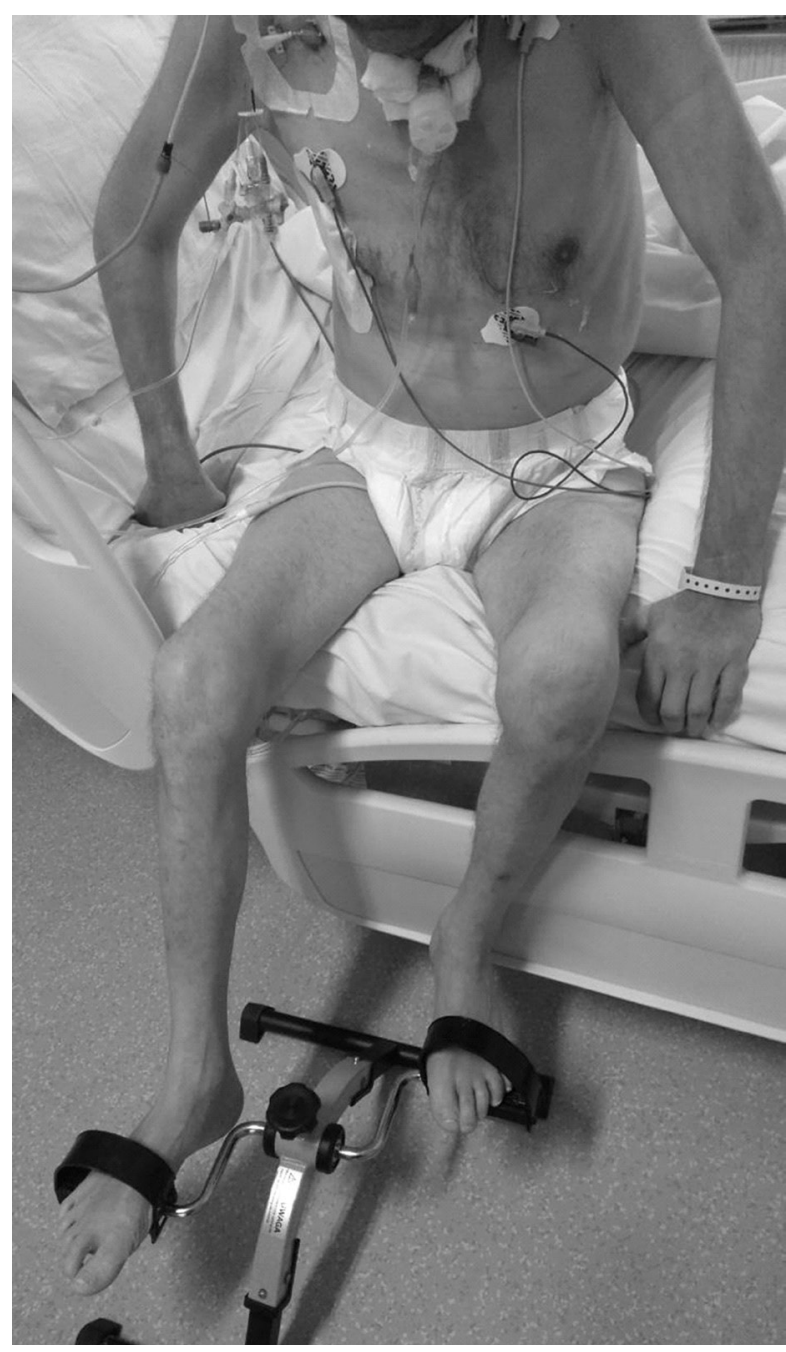

Figure 1. Training with a bedside cycle ergometer (informed consent to publication was obtained from patient)

ual work by stimulating the abdominal muscles to use indirect work on the respiratory system because after thoracic surgery, direct work on the surface of the ribs and sternum was impossible.

Rehabilitation was carried out 6 days a week continuing the plan of the program. On the $19^{\text {th }}$ day, the patient was in logical contact without sedation and was disconnected from the respirator. He remained on his own breathing through the tracheotomy tube. The next verticalizations took place in full circulatory and respiratory capacity, with an oxygen saturation of 94-99\%. The bone window created in the thorax wall allowed for open healing of the abscess (Figure 2). In addition to parenteral feeding through a central port, an easily digestible diet rich in protein was introduced. The daily verticalization of the patient from the 14th day of hospitalization prevented the development of contractures of the short muscles of the feet and Achilles tendons. Verticalization of 


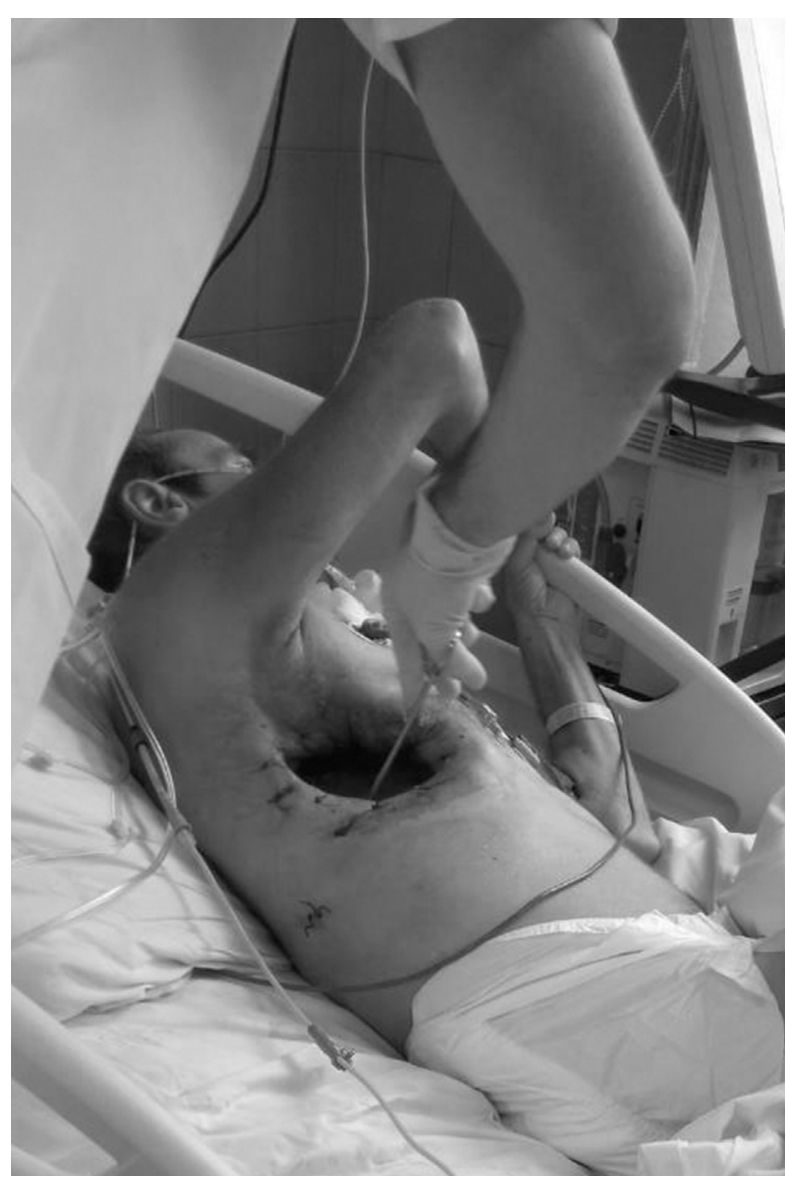

Figure 2. Dressing change (informed consent to publication was obtained from patient)

the patient in stages forced him to be active and involved. In the first period, he stabilized his feet, knee joints, pelvis, and shoulder girdle. At the end of the rehabilitation process, after applying appropriate knee joint fixation, the patient stood independently aided only by support at one location (on the sacral bone). In the afternoon, the patient performed simple self-assisted exercises of the upper limbs in a sitting position. Rehabilitation of the critically ill patient was conducted under constant monitoring of the bodily systems: blood pressure, ECG, saturation, and capnometry. On the $26^{\text {th }}$ day, a cardiopulmonary and respiratory capable patient with passive oxygen therapy was transferred to the Department of Thoracic Surgery where he stayed for the next 10 days.

After the fenestration was closed, pleural sterilization was performed through a drain with an iodine solution and a dressing change. The chest was asymmetrical. The rehabilitation process was continued and exercises with blue Thera-band were introduced. The patient was educated about the purposefulness of carrying out resistance exercises with tools such as a water bottle or Trifflo apparatus. The correct performance of these exercises aided in the evacuation of bronchial tree secretions. From the very first day in the Thoracic Surgery department, the most important thing in the rehabilitation process was the patient's independent activity. The exercises in the ICU were initially assisted on by a therapist. As the patient's muscle strength and education improved, they began to be initiated by the patient himself and were performed several times a day after receiving proper instructions from the physiotherapist. An increase in the strength of the respiratory muscles was observed in the following days. There was also a significant increase in chest circumference and a reduction in dyspneic episodes during resisted breathing exercises. Building trust and a sense of security in a critically ill patient is an important foundational step that initiates good therapy perspectives, especially when taking into account the long duration of rehabilitation at all stages of treatment.

The patient was discharged home from the Clinical Thoracic Surgery Department after 36 days in the Provincial Clinical Hospital - Center for Lung Diseases in a stable condition. He was fully capable and independent. The patient remained in the care of the Thoracic Surgery Outpatient Clinic where he had regular follow-ups.

\section{Discussion}

Interdisciplinary rehabilitation undertaken in the ICU is an indispensable element of the holistic treatment process of patients. According to Parker et al., programmed physiotherapy contributes to a reduction in the occurrence of possible disorders acquired by surgical patients in the ICU [1] Dhand reports that polyneuropathy, myopathy, and prolonged neuromuscular blockade are now considered a frequent cause of newly acquired disease syndromes in these patients [2]. Early rehabilitation is associated with increased functional capacity and muscle strength in critically ill patients. The cyclical nature of verticalization affects the patient's perceived quality of life. In addition, Chapman et al. demonstrated that keeping the patient in a semi-high position not only protects him from aspiration of offending agents in the alimentary tract, but also protects against ventilator-associated pneumonia [3]. Ibáñez et al. presented similar conclusions emphasizing the significant impact of early rehabilitation in critically ill patients which reduced the time of their hospitalization in the ICU [4]. Other studies have shown that early physical mobilization 
can be performed in critically ill patients even when they require mechanical ventilation thanks to movement patterns based on Proprioceptive Neuromuscular Facilitation (PNF) [5]. It is emphasized that actions to improve the state of critically ill patients should be carried out while observing the results of monitoring systems including blood pressure, ECG, and oxygen saturation [6]. It has been shown that an increase in respiratory function is associated with the use of resistance and strengthening exercises which influence the increase in the efficiency of the critically ill organism [7]. The effectiveness of respiratory rehabilitation based on the elements of the PNF method to increase respiratory functions was also demonstrated by authors who carried out research using resistance and strengthening respiratory exercises in neurological patients in ICUs $[8,9]$. Park et al. recommended indirect work on the respiratory system when direct work on the ribs is impossible so that the abdominal muscles are stimulated [10]. It was also demonstrated that bedside cycling can improve rehabilitation in critically ill patients as it ensures low-intensity movement and introduces activity to patients after pneumonectomy which affects the individual improvement of the body's capacity [11]. Our paper joins the growing collection of literature that suggests that early training cycles on a bedside bike can safely be carried out with critically ill patients. Camargo Pires-Neto et al. confirmed that a single 20-minute cycling session commenced within the first 72 hours showed no safety concern as long as patients received low-dose infusions of a vasoactive drug to limit the increase in cardiac output [12]. Cycling specifically targets the legs and requires movements that involve hip flexion which is important because these are parts of the body that are most susceptible to muscle atrophy and weakness when lying in bed [13]. Maintaining muscular strength may improve functional outcome as both paresis acquired at ICU and decreased muscle strength were independently associated with poor hospital results [14]. Early rehabilitation had a significant impact on the functional state, muscle strength, mechanical ventilation time, gait capacity on discharge, and overall quality of life [15].

The analysis of the material as well as the authors' own experiences allow for the formulation of several conclusions regarding early thoracic-surgical rehabilitation of the patient at the ICU. The main goal of rehabilitation is to prevent complications resulting from immobilization by instilling early and functional mobilization of the patient which shortens hospitalization and accelerates the return of functional capacity by improving the patient's quality of life.

\section{Conflict of interest}

None declared.

\section{References:}

1. Parker A, Sricharoenchai T, Needham DM. Early rehabilitation in the intensive care unit: preventing physical and mental health impairments. Curr Phys Med Rehabil Rep. 2013; 1(4): 307-314, doi: 10.1007/s40141-013-0027-9, indexed in Pubmed: 24436844.

2. Dhand UK. Clinical approach to the weak patient in the intensive care unit. Respir Care. 2006; 51(9): 1024-40; discussion 1040, indexed in Pubmed: 16934166.

3. Chapman AR, Litton EL. Primary prevention in the intensive care unit: a prospective single-centre study of the risk factors for invasive pneumococcal disease. Anaesth Intensive Care. 2017; 45(4): 448-452, doi: 10.1177/0310057X1704500406, indexed in Pubmed: 28673213.

4. Ibáñez J, Peñafiel A, Raurich JM, et al. Gastroesophageal reflux in intubated patients receiving enteral nutrition: effect of supine and semirecumbent positions. JPEN J Parenter Enteral Nutr. 1992; 16(5): 419-422, doi: 10.1177/0148607192016005419, indexed in Pubmed: 1433774.

5. Conceição TM, Gonzáles AI, Figueiredo FC, et al. Safety criteria to start early mobilization in intensive care units. Systematic review. Rev Bras Ter Intensiva. 2017; 29(4): 509-519, doi: 10.5935/0103-507X.20170076, indexed in Pubmed: 29340541.

6. Arias-Fernández P, Romero-Martin M, Gómez-Salgado J, et al. Rehabilitation and early mobilization in the critical patient: systematic review. J Phys Ther Sci. 2018; 30(9): 1193-1201, doi: 10.1589/ipts.30.1193, indexed in Pubmed: $\underline{30214124}$.

7. Liaw MY, Lin MC, Cheng PT, et al. Resistive inspiratory muscle training: its effectiveness in patients with acute complete cervical cord injury. Arch Phys Med Rehabil. 2000; 81(6): 752-756, doi: 10.1016/s0003-9993(00)90106-0, indexed in Pubmed: 10857519.

8. Seo K, Cho M. The effects on the pulmonary function of normal adults proprioceptive neuromuscular facilitation respiration pattern exercise. J Phys Ther Sci. 2014; 26(10): 1579-1582, doi: 10.1589/jpts.26.1579, indexed in Pubmed: 25364117.

9. Adler J, Malone D. Early mobilization in the intensive care unit: a systematic review. Cardiopulm Phys Ther J. 2012; 23(1): 5-13, indexed in Pubmed: 22807649.

10. Park SY, Park IK, Hwang Y, et al. Immediate postoperative care in the general thoracic ward is safe for low-risk patients after lobectomy for lung cancer. Korean J Thorac Cardiovasc Surg. 2011; 44(3): 229-235, doi: 10.5090/kjtcs.2011.44.3.229, indexed in Pubmed: 22263157.

11. Needham DM, Truong AD, Fan E. Technology to enhance physical rehabilitation of critically ill patients. Crit Care Med. 2009; 37(10 Suppl): S436-S441, doi: 10.1097/CCM.0b013e3181b6fa29, indexed in Pubmed: 20046132.

12. Camargo Pires-Neto R, Fogaça Kawaguchi YM, Sayuri Hirota A, et al. Very early passive cycling exercise in mechanically ventilated critically ill patients: physiological and safety aspects--a case series. PLoS One. 2013; 8(9): e74182, doi: 10.1371/journal.pone.0074182, indexed in Pubmed: 24040200.

13. Iannuzzi-Sucich M, Prestwood KM, Kenny AM. Prevalence of sarcopenia and predictors of skeletal muscle mass in healthy, older men and women. J Gerontol A Biol Sci Med Sci. 2002; 57(12): M772-M777, doi: 10.1093/gerona/57.12.m772, indexed in Pubmed: 12456735.

14. Arias-Fernández P, Romero-Martin M, Gómez-Salgado J, et al. Rehabilitation and early mobilization in the critical patient: systematic review. J Phys Ther Sci. 2018; 30(9): 1193-1201, doi: 10.1589/jpts.30.1193, indexed in Pubmed: 30214124.

15. Yeampattanaporn O, Mekhora K, Jalayondeja W, et al. Immeand range of motion in chronic neck pain. J Med Assoc Thai. 2014; 97 Suppl 7: S55-S59, indexed in Pubmed: 25141528. 